\title{
Neon abundances in three Wolf-Rayet stars observed with the ISO Short Wavelength Spectrometer
}

\author{
Patrick W. Morris ${ }^{1}$ and Karel A. van der Hucht \\ SRON, Sorbonnelaan 2, NL-3584 CA Utrecht, the Netherlands \\ Allan J. Willis, Luc Dessart, and Paul A. Crowther \\ Department of Physics \& Astronomy, University College London, \\ Gower St, London WC1E 6BT, UK
}

Peredur M. Williams

Royal Observatory, Blackford Hill, Edinburgh EH9 3HJ, UK

\section{Introduction}

We are motivated to study the $\mathrm{Ne} / \mathrm{He}$ abundance ratio at the surfaces of WolfRayet stars by evolutionary models, which have predicted enhancements by more than an order of magnitude over cosmic levels during the WC stage (e.g., Maeder 1991). This should occur early in the WC phase, as core-hydrogen is exhausted and the CNO bi-cycle gives way to the following chain of reactions for the Galactic stars:

$$
{ }^{14} \mathrm{~N}(\alpha, \gamma) \longrightarrow{ }^{18} \mathrm{~F}\left(\beta^{+}, \nu\right) \longrightarrow{ }^{18} \mathrm{O}(\alpha, \gamma) \longrightarrow{ }^{22} \mathrm{Ne}(\alpha, n) \longrightarrow{ }^{25} \mathrm{Mg}
$$

The latest evolutionary models, which include the effects of interior mixing due to stellar rotation, indicate that the enhancement may be somewhat more gradual, but that the $\mathrm{Ne} / \mathrm{He}$ ratio is essentially unchanged in its peak value, coinciding with complete H-depletion (e.g., Meynet, these Proceedings).

\section{Latest ISO results}

The first WC star to be studied in detail, $\gamma^{2}$ Vel (WR 11, WC8+O8.5III), showed only a minor enhancement of $\mathrm{Ne} / \mathrm{He}$ (Barlow et al. 1988). This was the only WC star for which an IRAS-LRS spectrum was available to measure the [NeIII] $15.55 \mu \mathrm{m}$ line emission. With ISO, $\gamma^{2}$ Vel and several other WR stars have been observed using the short wavelength spectrometer (sws). The reader is referred to Figure 4 in the review by A. Willis (these Proceedings) for a montage of several WR star spectra over the $12-17 \mu \mathrm{m}$ range that would include the lines of [NeII] $12.81 \mu \mathrm{m}$, [Nev] $14.32 \mu \mathrm{m}$, and [NeIII] $15.55 \mu \mathrm{m}$.

The sws and ground-based spectrum of WR $146(\mathrm{WC} 6+\mathrm{O})$ has been studied by Willis et al. (1997), who found $\mathrm{Ne} / \mathrm{He}$ to be much closer to the evolutionary

\footnotetext{
${ }^{1}$ Present address: Astronomical Institute, University of Amsterdam, the Netherlands
} 
predictions. Subsequently, Morris et al. (1998) presented preliminary results for WR 135 (WC8) and WR 147 (WN8(h)+B0.5V), and re-assessed the results for $\gamma^{2}$ Vel in light of the new distance established by Hipparcos (van der Hucht et al. 1997) and the mass-loss rate estimates by St-Louis et al. (1988) and Stevens et al. (1996).

\subsection{WR 135 (WC8)}

The Ne/He ratio of WR 135 is roughly a factor four higher than expected for an environment where $\mathrm{H} / \mathrm{He}=0.0$ and $\mathrm{C} / \mathrm{He}=0.13$, but this is not as much as the evolutionary models predict. There is some hint of [Mgv] $13.54 \mu \mathrm{m}$ emission, which could indicate that $\alpha$ capture by ${ }^{22} \mathrm{Ne}$ has occured. We do not notice magnetic dipole transtions of [Mgv] $5.608 \mu \mathrm{m}$ or [MgIv] $4.488 \mu \mathrm{m}$.

\subsection{WR 11 (WC8+O8.5III)}

The Ne-line fluxes at $12.81 \mu \mathrm{m}$ and $15.55 \mu \mathrm{m}$ are in good agreement with the values quoted by Barlow et al. (1988), but the stellar distance is now known to be nearly a factor of two closer based on Hipparcos observations. This by itself does not influence the results much, since the ion fraction $\gamma_{i}$ scales as $d^{2} / \dot{M}^{3 / 2}$, and $\dot{M}$ scales as $d^{3 / 2}$. However, much lower mass-loss rates are given by polarimetery studies by St-Louis et al. (1988) and X-ray observations by Stevens et al. (1996), roughly three times lower than estimated by Barlow et al. (1988) from the radio free-free continuum. Using an average $\dot{M}=1.3 \times 10^{-4} \mathrm{M}_{\odot} \mathrm{yr}^{-1}$ and $\mathrm{C} / \mathrm{He}=0.14$ from recombination analysis of the sws lines, a lower value of $\mathrm{Ne} / \mathrm{He} \geq 6.3 \times 10^{-3}$ is obtained, a factor of $\sim 10$ higher than a cosmic environment where $\mathrm{H} / \mathrm{He}=0.0$ and $\mathrm{C} / \mathrm{He}=0.14$.

\subsection{WR 147 (WN8(h)+B0.5V)}

The sws spectrum of WR 147 shows very strong [NeIII] $15.55 \mu \mathrm{m}$ emission, and no [NeII] $12.81 \mu \mathrm{m}$ line (e.g., Morris et al. 1998). Nonetheless, Ne in the ratio with He should be normal, since no net Ne production occurs during the CNO bicycle. To check $\mathrm{Ne} / \mathrm{He}$, we relied on the mass-loss rate estimated by Hamann et al. (1995) from optical spectroscopy. Their value was re-scaled to the distance and interstellar reddening given by Churchwell et al. (1992), using $\mathrm{M}_{v}=v-$ $\mathrm{DM}-A_{v}, A_{v}=4.1 E_{b-v}$, and equations (4) and (5) in Hamann et al. (1993). This leads to a lower limit of $\mathrm{Ne} / \mathrm{He} \geq 1.0 \times 10^{-3}$, which is a factor of 2 or 3 higher than expected for an environement where $\mathrm{H} / \mathrm{He}$ is somewhere between 0.0 and 0.4 . With respect to levels predicted for WC stars, the enhancement is hardly that, but it is significant for a WN star, and may indicate some exposure of core-processed material via rotational mixing, as predicted to occur in WR stars (Meynet, these Proceedings).

\section{The $\dot{M}$ problem}

As mentioned, the Ne-ion fraction scales as $\dot{M}^{-3 / 2}$ in the two-level atom, massloss normalizing method by Barlow et al. (1988). Among published results using spherical, homogeneous wind models, differences by a factor of three in the value of $\dot{M}$ are not unusual due to distance and (to a lesser extent) reddening 
uncertainties, and the inclusion of line-blanketing by metals. More important may be the assumptions on geometry and homegeneity of the wind. It is known that clumping may lower the mass-loss rate by factors of two to three, which would serve to increase the ion fractions in the clumps, according to the inverse scaling with $\dot{M}$. This is emphasized by the case of WR 11 , and might rectify the low $\mathrm{Ne} / \mathrm{He}$ abundance ratio in WR 135 . It would normally, however, exacerbate an already unexpected (albeit minor at the moment) over-abundance in the WN8 star of WR 147. But the system is highly reddened at $A_{V} \simeq 11.5 \mathrm{mag}$ (Churchwell et al. 1992), and the foreground extinction properties are probably anomylous, contributing to the difficulty in arriving at a clean spectroscopic solution for the physical parameters of the system. These issues are addressed in papers in preparation, where we will present the ISO-SWS spectra of WR 11, WR 135, and WR 147 in detail.

\section{References}

Barlow, M.J., Roche, P.F., Aitken, D.K. 1988, MNRAS 232, 821

Churchwell, E., Bieging, J.H., van der Hucht, K.A., Williams, P.M., Spoelstra, T.A.Th., Abbott, D.C. 1992, ApJ 393, 329

Hamann, W.-R., Koesterke, L., Wessolowski, U. 1993, A\&A 274, 397

Hamann, W.-R., Koesterke, L., Wessolowski, U. 1995, A\&A 299, 151

van der Hucht, K. A., Schrijver, H., Stenholm, B., et al. 1997, New Astron. 2, 245

Maeder, A. 1991, A\&A 242, 93

Morris, P.W., van der Hucht, K.A., Willis, A.J., Williams, P.M. 1998, in: L.B.F.M. Waters, C. Waelkens, K.A. van der Hucht \& P.A. Zaal (eds.), ISO's View on Stellar Evolution, ApSS 255, 157

St-Louis, N., Moffat, A.F.J., Drissen, L., et al. 1988, ApJ 330, 286

Stevens, I.R., Cocoran, M.F., Willis, A.J., et al. 1996, MNRAS 283, 589

Willis, A.J., Dessart, L., Crowther, P.A., Morris, P.W., Maeder, A., Conti, P.S., van der Hucht, K.A. 1997, MNRAS 290, 371

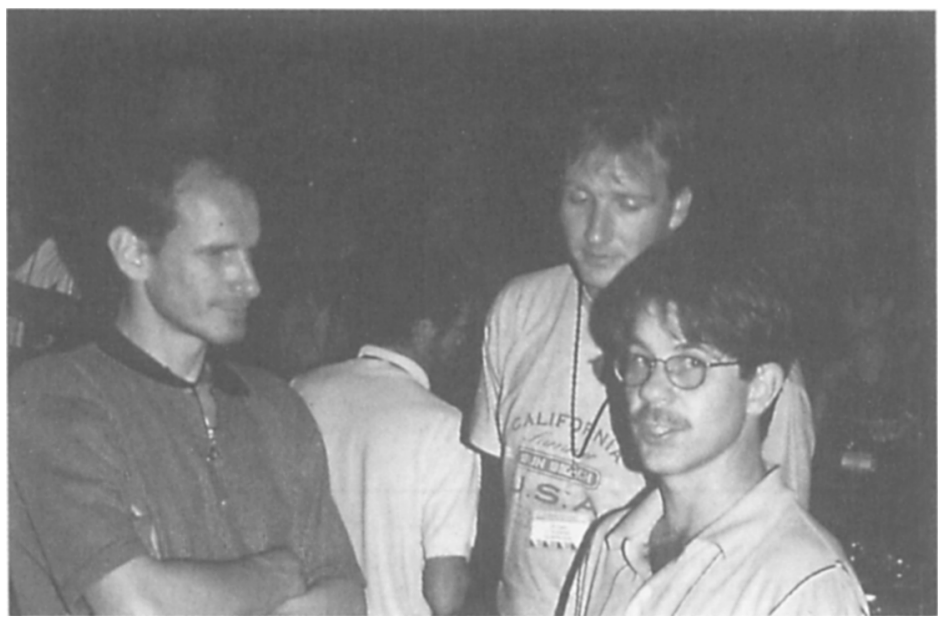

